\title{
On the Latent Roots of Certain Matrices.
}

\author{
By A. C. Aitren. \\ (Received 14th May 1927. Read 4th June 1927.)
}

\section{§1. INTRODUCTORY.}

In the present note certain known theorems on the latent roots of matrices are deduced from the fundamental theorem that a matrix $A$ can be expressed in the form $P Q P^{-1}$, where $P$ is non-singular and $Q$ has zero elements everywhere to the left of the principal diagonal, and the latent roots of $A$ in the diagonal. [The presence or absence of non-zero elements to the right of the diagonal is known to depend on the nature of the "elementary divisors" of the "characteristic determinant" of $A$, but in what follows these will not concern us.]

We shall here consider three kinds of matrices related in prescribed ways to a given matrix $A$, or to a given set of matrices $A_{j}, j=1,2, \ldots m$. In each case considered the following is the type of theorem to be established:

The related matrix of the product of any number of given matrices is identical with the product of the related matrices of the given matrices.

\section{$\S 2$. Compound Matrices.}

By $A^{(m)}$, the $m^{\text {th }}$ compound of $A$, we denote the matrix which has for its elements minors of order $m$ of the determinant of $A,|A|$, priority of location of these in $A^{(m)}$ being decided by priority of rows and columns in $A$; in other words, the matrix of the $m^{\text {th }}$ compound of $|A|$ as usually defined in the theory of determinants. We then state:

The $m^{\text {th }}$ compound of the product of any number of matrices is identical with the product of the $m^{\text {th }}$ compounds of those matrices.

This theorem is really due to Cauchy, ${ }^{1}$ who considered the $m^{\text {th }}$ "derived system," as he called it, of the product of two determinants; it is an immediate consequence of the Binet-Cauchy theorem on the multiplication of rectangular arrays and indeed, as given by Cauchy, is almost an alternative statement of that wellknown theorem. The corresponding result for powers of a single matrix is evident; in particular then we have

$$
A^{(m)}=P^{(m)} Q^{(m)}\left(P^{-1}\right)^{(m)}=P^{(m)} Q^{(m)}\left(P^{(m)}\right)^{-1} .
$$

I See Muir's History of the Theory of Determinants, Vol. I, p. 121. 
Now $\left|P^{(m)}\right|$, by Sylvester's theorem on compound determinants, is a power of $|P|$, and so $P^{(m)}$ is a non-singular matrix. Also $Q^{(m)}$, by its mode of formation, is seen to have zero elements everywhere to the left of the principal diagonal, and $m$-ary products of the latent roots of $A$ in the diagonal. So we have Rados' 1 theorem:

The latent roots of the $m^{\text {th }}$ compound of $A$ are the $m$-ary products of the latent roots of $A$.

\section{§3. Multilinear Matrices.}

Consider again $m$ different sets of $n$ variables, all distinct, taking with each set $n$ homogeneous linear functions of the variables, the matrix of the $j^{\text {th }}$ set of functions being $A_{j}, j=1,2, \ldots m$. Let us form in all possible ways products of $m$ of these linear functions, one from each set. We thus obtain $n^{m}$ functions, which we may regard as linear in the $n^{m} m$-ary product variables. The matrix of these will be called the multilinear matrix of the $A_{j}$ 's and will be denoted by $M\left[A_{j}\right]$.

To fix ideas, let us take two sets of linear functions in three variables,

$$
\left\{\begin{array} { l } 
{ a _ { 1 } x + a _ { 2 } y + a _ { 3 } z } \\
{ b _ { 1 } x + b _ { 2 } y + b _ { 3 } z } \\
{ c _ { 1 } x + c _ { 2 } y + c _ { 3 } z }
\end{array} \quad \text { and } \quad \left\{\begin{array}{l}
a_{1} \xi+a_{2} \eta+a_{3} \zeta \\
\beta_{1} \xi+\beta_{2} \eta+\beta_{3} \zeta \\
\gamma_{1} \xi+\gamma_{2} \eta+\gamma_{3} \zeta
\end{array}\right.\right.
$$

Then the bilinear matrix of $\left[a_{1} b_{2} c_{3}\right]$ and $\left[\alpha_{1} \beta_{2} \gamma_{3}\right]$, say

$$
M\left[\left[a_{1} b_{2} c_{3}\right],\left[a_{1} \beta_{2} \gamma_{3}\right]\right],
$$

will be sufficiently indicated by its first row and column, namely

and $\begin{array}{llllllllll}a_{1} a_{1} & a_{1} a_{2} & a_{1} a_{3} & a_{2} a_{1} & a_{2} \alpha_{2} & a_{2} a_{3} & a_{3} \alpha_{2} & a_{3} a_{2} & a_{3} a_{3} \\ a_{1} a_{1} & a_{1} \beta_{1} & a_{1} \gamma_{1} & b_{1} \alpha_{1} & b_{1} \beta_{1} & b_{1} \gamma_{1} & c_{1} \alpha_{1} & c_{1} \beta_{1} & c_{1} \gamma_{1}\end{array}$

it being noted that the combination of elements from rows of the original matrices is made in the same order as from columns.

Now premultiply $M\left[\left[l_{1} m_{2} n_{3}\right],\left[\lambda_{1} \mu_{2} \nu_{3}\right]\right]$ by $M\left[\left[a_{1} b_{2} c_{3}\right],\left[a_{1} \beta_{2} \gamma_{3}\right]\right]$. The leading element in the product matrix is

$$
\begin{aligned}
& a_{1} a_{1} l_{1} \lambda_{1}+a_{1} a_{2} l_{1} \mu_{1}+a_{1} a_{3} l_{1} \nu_{1} \ldots+a_{3} a_{3} n_{1} \nu_{1} \\
= & \left(a_{1} l_{1}+a_{2} m_{1}+a_{3} n_{1}\right)\left(\alpha_{1} \lambda_{1}+a_{1} \mu_{1}+a_{3} \nu_{1}\right) .
\end{aligned}
$$

But this is the leading element in

$$
M\left[\left[a_{1} b_{2} c_{3}\right]\left[l_{1} m_{2} n_{3}\right],\left[\alpha_{1} \beta_{2} \gamma_{3}\right]\left[\lambda_{1} \mu_{2} \nu_{3}\right]\right] .
$$

1 Zur Theorie der Adjungirten Substitutionen. Math. Ann. 48 (1897), 417-424. 
A similar result may be shown to hold for every element, and indeed for general multilinear matrices we have without difficulty

$$
M\left[A_{j}\right] M\left[B_{j}\right] \ldots M\left[K_{j}\right]=M\left[A_{j} B_{j} \ldots K_{j}\right]
$$

or: The product of any number of multilinear matrices of different sets of matrices $A_{j}, B_{j}, \ldots K_{j}$ is identical with the multilinear matrix of the set $A_{j} B_{j} \ldots K_{j}$.

By making all the sets the same we deduce as before the corresponding result for powers of multilinear matrices. Thus

$$
\left\{M\left[A_{j}\right]\right\}^{p}=M\left[A_{j}^{p}\right]
$$

Now suppose $A_{j}=P_{j} Q_{j} P_{j}=1$, the latent roots of $A_{j}$ being $\lambda_{1 j}, \lambda_{2 j}, \ldots \lambda_{n j}$. Then we must have

$$
\begin{aligned}
M\left[A_{j}\right] & =M\left[P_{j}\right] M\left[Q_{j}\right] M\left[P_{j}^{-1}\right] \\
& =M\left[P_{j}\right] M\left[Q_{j}\right]\left\{M\left[P_{j}\right]\right\}^{-1}
\end{aligned}
$$

But $M\left[Q_{j}\right]$, from its mode of formation, will be seen to have zeros everywhere to the left of the principal diagonal, and in the diagonal $m$-ary products of $\lambda$ 's, taken one from each set. Hence we have:

The latent roots of the multilinear matrix of $m$ given matrices are the $m$-ary products of the latent roots of the matrices, taken one from each matrix.

The particular case of this where all the matrices are identical has been given by W. Burnside. ${ }^{1}$

\section{§4. Schläflian Matrices.}

If in the previous section we let not only all the matrices but also all the variables of the linear functions be made identical with those of one set, and then collect terms in the same $m$-ary product variables, which will appear more than once with factors permuted, we now obtain ${ }^{n} H_{m}$ functions in ${ }^{n} H_{m}$ variables. The matrix of these will be called the $m^{\text {th }}$ Schläflian of $A$. [Its determinant was shown by

1 On the characteristic equations of certain linear substitutions. Quart. Journ. Math. 33 (1902), 80-84. 
Schläfli ${ }^{1}$ to be a power of $|A|$.] For example the second Schläflian of $\left[a_{1} b_{2} c_{3}\right]$ is

$\left[\begin{array}{cccccc}a_{1}{ }^{2} & a_{2}{ }^{2} & a_{3}{ }^{2} & 2 a_{1} a_{2} & 2 a_{1} a_{3} & 2 a_{2} a_{3} \\ b_{1}{ }^{2} & b_{2}{ }^{2} & b_{3}{ }^{2} & 2 b_{1} b_{2} & 2 b_{1} b_{3} & 2 b_{2} b_{3} \\ c_{1}{ }^{2} & c_{2}{ }^{2} & c_{3}{ }^{2} & 2 c_{1} c_{2} & 2 c_{1} c_{3} & 2 c_{2} c_{3} \\ a_{1} b_{1} & a_{2} b_{2} & a_{3} b_{3} & a_{1} b_{2}+a_{2} b_{1} & a_{1} b_{3}+a_{3} b_{1} & a_{2} b_{3}+a_{3} b_{2} \\ a_{1} c_{1} & a_{2} c_{2} & a_{3} c_{3} & a_{1} c_{2}+a_{2} c_{1} & a_{1} c_{3}+a_{3} c_{1} & a_{2} c_{3}+a_{3} c_{2} \\ b_{1} c_{1} & b_{2} c_{2} & b_{3} c_{3} & b_{1} c_{2}+b_{2} c_{1} & b_{1} c_{3}+b_{3} c_{1} & b_{2} c_{3}+b_{3} c_{2}\end{array}\right]$.

By proceeding exactly as in $\S 3$ we have without difficulty:

The $m^{\text {th }}$ Schläfian of the product of any number of matrices is identical with the product of the $m^{\text {th }}$ Schlaflians of those matrices.

Analogous theorems flow as before for Schlätlians of powers of a matrix, and by examining the form of the $m^{\text {th }}$ Schläflian of $Q$ we have Franklin's ${ }^{2}$ theorem:

The latent roots of the $m^{\text {th }}$ Schläflian of a given matrix are the $m$-ary powers and products of the latent roots of the matrix.

For example the second Schlällian of

$\left[\begin{array}{ccc}\lambda_{1} & . & . \\ 0 & \lambda_{2} & . \\ 0 & 0 & \lambda_{3}\end{array}\right]$ is $\left[\begin{array}{cccccc}\lambda_{1}{ }^{2} & . & . & . & . & . \\ 0 & \lambda_{2}{ }^{2} & . & . & . & . \\ 0 & 0 & \lambda_{3}{ }^{2} & . & . & . \\ 0 & 0 & 0 & \lambda_{1} \lambda_{2} & . & . \\ 0 & 0 & 0 & 0 & \lambda_{1} \lambda_{3} & . \\ 0 & 0 & 0 & 0 & 0 & \lambda_{2} \lambda_{3}\end{array}\right]$,

the elements above the diagonals being left undetermined.

It may be remarked that the result on latent roots of multilinear matrices is included in a rather more general theorem of Metzler, ${ }^{3}$ in which the different sets of linear functions in $\$ 3$ involve different numbers of variables. On the other hand the more general result could be deduced from that of $\S 3$ by taking the elements of a certain number of the last rows and columns of $A_{j}$ to be all zero, so that each matrix may have some zero latent roots. From our present point of view, however, it is not the properties of the latent roots, which are much less fundamental than the elementary divisors, but the indifference as to the order of the operations of forming products, compounds, multilinear or Schläflian matrices that is regarded as having importance.

${ }^{1}$ See Muir's History of the Theory of Determinants, Vol. II, pp. 52-53.

2 Note on Induced Linear Substitutions. Amer. Journ. Math. 16 (1894), pp. 205-206.

${ }^{3}$ A Determinantal Equation whose Roots are the Product of the Roots of Given Determinantal Equations. Proc. Roy. Soc. Edin. 38 (1917), 57-60. 